\title{
Saving for Retirement at Work: Views of Voters Ages 25+ Annotated Questionnaire
}

Screening criteria:

Ages $25+$

Registered to vote

This survey was fielded by SSRS in English and Spanish to the SSRS probability-based panel from September 22, 2021 to September 29, 2021. Ninety-three percent of the respondents completed the survey online; seven percent completed the survey by phone.

Results were weighted to represent adults ages $25+$ who are registered to vote.

All percentages shown below are weighted. The base for each question is shown as an unweighted number. Percentages may not always sum to 100 due to rounding.

\section{Base Size: $n=1,010$ except where noted}

CS4_a. Could you please tell me if your age falls into one of these ranges?

\begin{tabular}{|l|c|}
\hline & $\%$ \\
\hline $25-44$ & 35 \\
\hline $45-64$ & 38 \\
\hline $65+$ & 27 \\
\hline
\end{tabular}

\section{SCREENER}

QS6. Which of the following best describes your current employment status? Are you...

\begin{tabular}{|l|c|}
\hline & $\%$ \\
\hline Self Employed (Net) & $\mathbf{9}$ \\
\hline Self-employed full-time & 4 \\
\hline Self-employed part-time & 4 \\
\hline Employed Full/Part-Time (Net) & $\mathbf{5 2}$ \\
\hline Employed full-time & 44 \\
\hline Employed part-time & 8 \\
\hline Retired and not working at all & 30 \\
\hline Unemployed and looking for work & 4 \\
\hline Not working and not looking for work (but not retired) & 6 \\
\hline Don't know/Refused/Decline/Web blank & $<1$ \\
\hline
\end{tabular}

https://doi.org/10.26419/res.00500.002 


\section{ATTITUDINAL QUESTIONS}

1. As you think about your finances in the future, how anxious do you feel about having enough money to live comfortably through your retirement years?

\begin{tabular}{|l|l|}
\hline & $\%$ \\
\hline Anxious (Net) & 63 \\
\hline Very anxious & 24 \\
\hline Somewhat anxious & 39 \\
\hline Not anxious (Net) & 37 \\
\hline Not very anxious & 24 \\
\hline Not anxious at all & 14 \\
\hline Don't know/Refused/Decline/Web blank & -- \\
\hline
\end{tabular}

2. How important is it for people to be able to save money for their retirement years while they are working?

\begin{tabular}{|l|c|}
\hline & $\%$ \\
\hline Important (Net) & $\mathbf{1 0 0}$ \\
\hline Very important & 93 \\
\hline Somewhat important & 7 \\
\hline Not important (Net) & $<1$ \\
\hline Not very important & $<1$ \\
\hline Not important at all & $<1$ \\
\hline Don't know/Refused/Decline/Web blank & -- \\
\hline
\end{tabular}


3. AGE 25-44: Given the amount of money you currently have saved, do you think you'll be able to save enough money for your retirement years?

(Base: Not retired and Age 25-44; $n=342$ )

\begin{tabular}{|l|c|}
\hline & \% \\
\hline Yes & 29 \\
\hline No & 45 \\
\hline Don't know & 26 \\
\hline Refused/Decline/Web blank & -- \\
\hline
\end{tabular}

AGE 45+: Given the amount of money you currently have saved, do you wish you had more money saved for your retirement years?

(Base: Not retired and Age 45+; $n=362$ )

\begin{tabular}{|l|c|}
\hline & $\%$ \\
\hline Yes & 81 \\
\hline No & 15 \\
\hline Don't know & 4 \\
\hline Refused/Decline/Web blank & -- \\
\hline
\end{tabular}

4. When it comes to planning and saving for retirement, would you say that you are ahead of schedule, on track, or behind schedule?

(Base: Not retired; $n=704$ )

\begin{tabular}{|l|c|}
\hline & \% \\
\hline Ahead of schedule & 9 \\
\hline On track & 31 \\
\hline Behind schedule & 60 \\
\hline Don't know/Refused/Decline/Web blank & -- \\
\hline
\end{tabular}




\section{RETIREMENT SAVINGS BEHAVIOR QUESTIONS}

QB1. Which, if any, of the following ways to save for retirement does your current employer provide?

A. An employer-funded traditional pension plan that supplies retirees with a monthly income, typically based on a formula of salary and years of service. [It is not a 401(k) plan].

(Base: Employed but not Self Employed; $n=500$ )

\begin{tabular}{|l|c|}
\hline & \% \\
\hline Yes & 28 \\
\hline No & 62 \\
\hline Don't know & 10 \\
\hline Refused/Decline/Web blank & -- \\
\hline
\end{tabular}

B. A workplace retirement savings plan such as a $401 \mathrm{k}, 403 \mathrm{~b}$, payroll deduction IRA, or other retirement savings plan that allows you to make contributions from your salary to an individual account set up in your name.

(Base: Employed but not Self Employed; $n=500$ )

\begin{tabular}{|l|c|}
\hline & $\%$ \\
\hline Yes & 77 \\
\hline No & 18 \\
\hline Don't know & 6 \\
\hline Refused/Decline/Web blank & -- \\
\hline
\end{tabular}

QB2. If your employer offered a way to save for retirement at work, how likely would you be to take advantage of it?*

(Base: Employer does not offer, don't know or refused; $n=91 *$ )

\begin{tabular}{|l|c|}
\hline & \% \\
\hline Likely (Net) & $\mathbf{9 1}$ \\
\hline Very likely & 54 \\
\hline Somewhat likely & 37 \\
\hline Not likely (Net) & $\mathbf{9}$ \\
\hline Not very likely & 6 \\
\hline Not at all likely & 3 \\
\hline Don't know/Refused/Decline/Web blank & -- \\
\hline
\end{tabular}

*Note: Results should be interpreted with caution due to small base of 91 respondents who qualified for this question. 
QB3. Are you making regular contributions to any of the following retirement savings tools?

a. A workplace retirement savings plan such as a $401 \mathrm{k}, 403 \mathrm{~b}$, payroll deduction IRA, or similar retirement savings plan offered by your employer

(Base: Not retired and current employer offers; $n=384$ )

\begin{tabular}{|l|c|}
\hline & $\%$ \\
\hline Yes & $85^{*}$ \\
\hline No & 14 \\
\hline Don't know/Refused/Decline/Web blank & $<1$ \\
\hline
\end{tabular}

*Note: This percentage ( $85 \%)$ is calculated only among those respondents whose employers offer a workplace retirement savings plan. This is different from the percentage included in the report of findings. The percentage in the report (65\%) is calculated among all employed respondents (excluding the self-employed) regardless of whether their employer offers a retirement savings plan.

b. A personal retirement savings plan outside of work such as an IRA

(Base: Not retired; $n=704$ )

\begin{tabular}{|l|c|}
\hline & $\%$ \\
\hline Yes & 29 \\
\hline No & 71 \\
\hline Don't know/Refused/Decline/Web blank & $<1$ \\
\hline
\end{tabular}

c. Something else to help you save for retirement

(Base: Not retired; $n=704$ )

\begin{tabular}{|l|c|}
\hline & $\%$ \\
\hline Yes & 44 \\
\hline No & 56 \\
\hline Don't know/Refused/Decline/Web blank & $<1$ \\
\hline
\end{tabular}

QB4. Thinking about the workplace retirement savings plan to which you are making contributions, how important is this retirement savings plan in helping you save for retirement?

(Base: Currently participating in a workplace plan; $n=323$ )

\begin{tabular}{|l|l|}
\hline & $\%$ \\
\hline Important (Net) & 96 \\
\hline Very important & 82 \\
\hline Somewhat important & 14 \\
\hline Not important (Net) & 4 \\
\hline Not too important & 4 \\
\hline Not important at all & -- \\
\hline Don't know/Refused/Decline/Web blank & -- \\
\hline
\end{tabular}




\section{CONCEPT DESCRIPTION QUESTIONS}

\section{(ASK ALL)}

QC1. Many workers in the U.S., especially those who work for small businesses, do not have a way to save for retirement at work. One way to help more workers save would be for Congress to pass a law that enables workers to save for retirement at work if their employer does not currently offer them a way to save. The program would offer workers control over their retirement savings by allowing them to choose whether or not to participate, decide how much to contribute, and let them take their account with them if they change jobs. Workers would save for retirement using money automatically taken out of their regular paycheck and deposited in a personal individual retirement account, commonly referred to as an IRA.

Do you support or oppose this program?

\begin{tabular}{|l|c|}
\hline & $\%$ \\
\hline Support (Net) & $\mathbf{9 1}$ \\
\hline Strongly support & 60 \\
\hline Somewhat support & 31 \\
\hline Oppose (Net) & $\mathbf{9}$ \\
\hline Somewhat oppose & 7 \\
\hline Strongly oppose & 2 \\
\hline Don't know/Refused/Decline/Web blank & -- \\
\hline
\end{tabular}




\section{(ASK ALL)}

QC2. [l'd like to read you a list of/Below is a list of] some features of a retirement savings program being considered for workers whose employers don't currently offer a retirement plan. For each feature, please indicate how important you think it would be for a retirement savings program to have that feature.

a. Portable - If you leave one job, you can take the money and account with you to your next job.

\begin{tabular}{|l|c|}
\hline & $\%$ \\
\hline Important (Net) & $\mathbf{9 9}$ \\
\hline Very important & 91 \\
\hline Somewhat important & 8 \\
\hline Not important (Net) & $\mathbf{1}$ \\
\hline Not very important & 1 \\
\hline Not important at all & $<1$ \\
\hline Don't know/Refused/Decline/Web blank & -- \\
\hline
\end{tabular}

b. Payroll deduction - Contributions are automatically deducted from each paycheck and deposited into a retirement account set up in your name.

\begin{tabular}{|l|c|}
\hline & $\%$ \\
\hline Important (Net) & 96 \\
\hline Very important & 71 \\
\hline Somewhat important & 25 \\
\hline Not important (Net) & 4 \\
\hline Not very important & 3 \\
\hline Not important at all & 1 \\
\hline Don't know/Refused/Decline/Web blank & $<1$ \\
\hline
\end{tabular}

c. Voluntary - Participants are not required to make any contributions

\begin{tabular}{|l|c|}
\hline & $\%$ \\
\hline Important (Net) & $\mathbf{9 0}$ \\
\hline Very important & 65 \\
\hline Somewhat important & 25 \\
\hline Not important (Net) & $\mathbf{1 0}$ \\
\hline Not very important & 6 \\
\hline Not important at all & 4 \\
\hline Don't know/Refused/Decline/Web blank & -- \\
\hline
\end{tabular}


d. Available to all employees who do not have a way to save for retirement at work, including those who work for small businesses

\begin{tabular}{|l|c|}
\hline & \% \\
\hline Important (Net) & $\mathbf{9 8}$ \\
\hline Very important & 79 \\
\hline Somewhat important & 19 \\
\hline Not important (Net) & $\mathbf{2}$ \\
\hline Not very important & 1 \\
\hline Not important at all & 1 \\
\hline Don't know/Refused/Decline/Web blank & -- \\
\hline
\end{tabular}

\section{(ASK ALL)}

QC3. Americans are more likely to save for retirement when they can do so out of their regular paycheck at work. Yet many workers do not have access to a workplace retirement savings plan, especially those that work at small businesses.

Thinking about this, do you agree or disagree with the following statement?

"Elected officials should support legislation that makes it easier for all workers to save for retirement out of their regular paycheck."

\begin{tabular}{|l|c|}
\hline & \% \\
\hline Agree (Net) & $\mathbf{9 2}$ \\
\hline Strongly agree & 69 \\
\hline Somewhat agree & 23 \\
\hline Disagree (Net) & $\mathbf{8}$ \\
\hline Somewhat disagree & 5 \\
\hline Strongly disagree & 3 \\
\hline Don't know/Refused/Decline/Web blank & -- \\
\hline
\end{tabular}




\section{DEMOGRAPHICS}

Transition Screen: These final questions are for demographic purposes only.

QS7. Are you currently employed in a federal, state, or local government job?

(Base: Employed Full Time or Part Time; $\mathbf{n = 6 0 2 )}$

\begin{tabular}{|l|c|}
\hline & $\%$ \\
\hline Yes & 19 \\
\hline No & 81 \\
\hline Don't know/Refused/Decline/Web blank & -- \\
\hline
\end{tabular}

\section{(MSTATUS)}

\section{(ASK ALL)}

D3. Which group describes your marital status?

\begin{tabular}{|l|c|}
\hline & \% \\
\hline Single, that is never married & 17 \\
\hline Single, living with a partner & 7 \\
\hline Married & 57 \\
\hline Separated & 2 \\
\hline Widowed & 7 \\
\hline Divorced & 11 \\
\hline Don't know/Refused/Decline/Web blank & -- \\
\hline
\end{tabular}

D4a. Are you or your spouse currently a member of AARP?

D4b. Are you or your partner currently a member of AARP?

D4c. Are you currently a member of AARP?

(Base: Age 40+ and married/living with partner OR Age 50+ and NOT married or living with partner; $n=683$ )

\begin{tabular}{|l|c|}
\hline & $\%$ \\
\hline Yes & 38 \\
\hline No & 62 \\
\hline Don't know/Refused/Decline/Web blank & -- \\
\hline
\end{tabular}


GENDER. (Z1) RECORD RESPONDENT'S SEX [DO NOT ASK]

\begin{tabular}{|l|c|}
\hline & $\%$ \\
\hline Male & 47 \\
\hline Female & 53 \\
\hline Another gender & -- \\
\hline Don't know/Refused & $<1$ \\
\hline
\end{tabular}

EDUC. (Z2) What is the highest level of school you have completed or the highest degree you have received?

\begin{tabular}{|l|c|}
\hline & $\%$ \\
\hline Less than high school (Grades 1-8 or no formal schooling) & $<1$ \\
\hline $\begin{array}{l}\text { High school incomplete (Grades 9-11 or Grade 12 with NO } \\
\text { diploma) }\end{array}$ & 26 \\
\hline $\begin{array}{l}\text { High school graduate (Grade 12 with diploma or GED certificate } \\
\text { or vocational, business technical or other training that did not } \\
\text { count toward a degree) }\end{array}$ & 16 \\
\hline Some college, no degree (includes some community college) & 11 \\
\hline Two year associate degree from a college or university & 20 \\
\hline $\begin{array}{l}\text { Four year college or university degree/Bachelor's degree (e.g., BS, } \\
\text { BA, AB) }\end{array}$ & 4 \\
\hline $\begin{array}{l}\text { Some postgraduate or professional schooling, no postgraduate } \\
\text { degree }\end{array}$ & 18 \\
\hline $\begin{array}{l}\text { Postgraduate or professional degree, including master's, } \\
\text { doctorate, medical or law degree (e.g., MA, MS, PhD, MD, JD) }\end{array}$ & -- \\
\hline Don't know/Refused/Decline/Web blank & \\
\hline
\end{tabular}

POLPARTY. (Z3) Generally speaking, do you usually think of yourself as...?

\begin{tabular}{|l|c|}
\hline & $\%$ \\
\hline A Republican & 31 \\
\hline A Democrat & 33 \\
\hline An Independent & 30 \\
\hline Something Else & 6 \\
\hline Don't know/Refused/Web blank & $<1$ \\
\hline
\end{tabular}


POLLEAN. (Z4) As of today, do you lean more to the Democratic Party or the Republican Party?

(Base: Independent/something else/don't know/refuse/blank; n=373)

\begin{tabular}{|l|c|}
\hline & $\%$ \\
\hline Democratic Party & 53 \\
\hline Republican Party & 44 \\
\hline Don't know/Refused/Web blank & 3 \\
\hline
\end{tabular}

POLVIEW. (Z5) Generally speaking, how would you describe your political views?

\begin{tabular}{|l|l|}
\hline & \% \\
\hline Conservative (Net) & $\mathbf{3 6}$ \\
\hline Very conservative & 12 \\
\hline Somewhat conservative & 24 \\
\hline Moderate & 35 \\
\hline Liberal (Net) & $\mathbf{2 9}$ \\
\hline Somewhat liberal & 18 \\
\hline Very liberal & 11 \\
\hline Don't know/Refused/Web blank & $<1$ \\
\hline
\end{tabular}

INC1. (Z6) What is your total annual household income from all sources, and before taxes?

\begin{tabular}{|c|c|}
\hline & $\%$ \\
\hline Less than $\$ 50,000$ (Net) & 39 \\
\hline Less than $\$ 15,000$ & 9 \\
\hline$\$ 15,000$ to less than $\$ 20,000$ & 9 \\
\hline$\$ 20,000$ to less than $\$ 25,000$ & $<1$ \\
\hline$\$ 25,000$ to less than $\$ 30,000$ & 4 \\
\hline$\$ 30,000$ to less than $\$ 40,000$ & 9 \\
\hline$\$ 40,000$ to less than $\$ 50,000$ & 7 \\
\hline Less than $\$ 50,000$ (Unspecified) & -- \\
\hline$\$ 50,000-<\$ 100,000$ (Net) & 34 \\
\hline$\$ 50,000$ to less than $\$ 75,000$ & 18 \\
\hline$\$ 75,000$ to less than $\$ 100,000$ & 16 \\
\hline$\$ 50,000$ but less than $\$ 100,000$ (Unspecified) & $<1$ \\
\hline$\$ 100,000$ and over (Net) & 26 \\
\hline$\$ 100,000$ to less than $\$ 150,000$ & 9 \\
\hline$\$ 150,000$ to less than $\$ 200,000$ & 6 \\
\hline$\$ 200,000$ to less than $\$ 250,000$ & 3 \\
\hline$\$ 250,000$ or more & 2 \\
\hline$\$ 100,000$ and over (Unspecified) & 5 \\
\hline Don't know/Refused/Decline/Web blank & 1 \\
\hline
\end{tabular}


29. Are you of Hispanic or Latino origin or descent?

SEE Z10.*

Z10. Do you consider yourself white, black or African American, Asian, Native American, Pacific Islander, mixed race or some other race?*

\begin{tabular}{|l|c|}
\hline & $\%$ \\
\hline White Non-Hispanic & 70 \\
\hline Black Non-Hispanic & 11 \\
\hline Hispanic & 10 \\
\hline Other & 8 \\
\hline Don't know/Refused/Web blank & -- \\
\hline
\end{tabular}

*Note: Results in this table reflect responses to Z9 and Z10. 